\title{
Morbidade da biópsia da próstata transretal guiada por ultrassonografia*
}

\author{
Morbidity of transrectal ultrasound guided prostate biopsy \\ Raphael Sandes Solha ${ }^{1}$, Sérgio Ajzen ${ }^{2}$, Harley De Nicola ${ }^{3}$, David Carlos Shigueoka ${ }^{4}$, \\ Hugo Alexandre Sócrates de Castro ${ }^{5}$
}

\begin{abstract}
Resumo Objetivo: Avaliar a incidência de complicações pós-procedimento nos pacientes submetidos a biópsia prostática transretal guiada por ultrassom no setor de intervenção do Departamento de Diagnóstico por Imagem da Escola Paulista de Medicina - Universidade Federal de São Paulo. Materiais e Métodos: Foram avaliados, via contato telefônico, 132 pacientes submetidos a biópsia de próstata transretal guiada por ultrassom no período de abril/2011 a junho/2011, seguindo o protocolo padrão do nosso setor. Resultados: As complicações pós-biópsia foram categorizadas em maiores e menores de acordo com a necessidade de avaliação médica adicional. Cinquenta e nove pacientes $(61,8 \%)$ apresentaram complicações, e desses, grande parte $(86,4 \%)$ apresentou sintomas leves e autolimitados, considerados menores. Oito pacientes $(8,2 \%)$ apresentaram complicações maiores, sendo que apenas um deles necessitou de tratamento sob regime de internação hospitalar. A retenção urinária foi a complicação maior mais incidente no nosso estudo. Conclusão: Corroborando outros estudos da literatura, nosso trabalho demonstrou baixa prevalência de complicações maiores após a biópsia prostática transretal.
\end{abstract}

Unitermos: Próstata; Biópsia; Morbidade; Ultrassonografia; Complicações.

\begin{abstract}
Objective: To evaluate the incidence of postprocedural complications in patients submitted to transrectal ultrasoundguided prostate biopsy at the Unit of Intervention, Department of Imaging Diagnosis of Escola Paulista de Medicina Universidade Federal de São Paulo. Materials and Methods: Telephone interviews were conducted with 132 patients who had undergone transrectal ultrasound-guided prostate biopsy in the period from April 2011 to June 2011, according to the institution's protocol. Results: Post-biopsy complications were categorized into two groups - minor and major complications, according to their need for further clinical evaluation. Complications were reported by 59 patients $(61.8 \%)$, most of them (86.4\%) with mild and self-limited symptoms, classified as minor complications. Eight patients (8.2\%) had major complications, one of which required in-hospital treatment. Urinary retention was the major and most common complication. Conclusion: The present study has demonstrated a low prevalence of major complications after transrectal prostate biopsy.
\end{abstract}

Keywords: Prostate; Biopsy; Morbidity; Ultrasonography; Complications.

Solha RS, Ajzen S, De Nicola H, Shigueoka DC, Castro HAS. Morbidade da biópsia da próstata transretal guiada por ultrassonografia. Radiol Bras. 2013 Mar/Abr;46(2):71-74.

\footnotetext{
* Trabalho realizado na Escola Paulista de Medicina - Universidade Federal de São Paulo (EPM-Unifesp), São Paulo, SP, Brasil.

1. Médico Residente do Departamento de Diagnóstico po Imagem da Escola Paulista de Medicina - Universidade Federal de São Paulo (EPM-Unifesp), São Paulo, SP, Brasil.

2. Professor Titular, Chefe do Departamento de Diagnóstico por Imagem da Escola Paulista de Medicina - Universidade Federal de São Paulo (EPM-Unifesp), São Paulo, SP, Brasil.

3. Doutor, Médico Colaborador do Departamento de Diagnóstico por Imagem da Escola Paulista de Medicina - Universidade Federal de São Paulo (EPM-Unifesp), São Paulo, SP, Brasil.

4. Professor Adjunto do Departamento de Diagnóstico po Imagem da Escola Paulista de Medicina - Universidade Federal de São Paulo (EPM-Unifesp), São Paulo, SP, Brasil.

5. Mestre, Chefe da Coordenadoria do Setor de Intervenção Não Vascular do Departamento de Diagnóstico por Imagem da Escola Paulista de Medicina - Universidade Federal de São Paulo (EPM-Unifesp), São Paulo, SP, Brasil.

Endereço para correspondência: Dr. Raphael Sandes Solha. Rua Barão do Triunfo, 1220, ap. 14, Brooklin Paulista. São Paulo, SP, Brasil, 04602-916. E-mail: r_solha@hotmail.com.

Recebido para publicação em 17/5/2012. Aceito, após revisã̃o, em 21/12/2012.
}

\section{INTRODUÇÃO}

O câncer de próstata representa a neoplasia mais comum entre os homens americanos, sendo a segunda maior causa de morte por câncer neste grupo populacional ${ }^{(1)}$. No Brasil, o câncer de próstata também é o mais comum entre os homens e, de acordo com o Instituto Nacional de Câncer, são estimados no Brasil cerca de 52.350 novos casos em $2010^{(2)}$. Acomete na sua grande maioria indivíduos com mais de 50 anos, sendo uma entidade heterogênea cujo espectro varia desde formas assintomáticas até formas rapidamente progressivas ${ }^{(3)}$. Apresenta taxa de mortalidade relativamente baixa, especialmente nos casos em que o diagnóstico é feito na fase inicial. Recentemente, esta taxa de mortalidade tem declinado, com índices de sobrevida em cinco anos chegando a 99\% em razão dos programas de rastreamento, da detecção precoce e das mudanças no estilo de vida ${ }^{(4)}$.

Apesar da evolução dos diversos métodos de diagnóstico por imagem na avaliação das doenças pélvicas, o câncer de próstata ainda tem seu diagnóstico confirmado pela avaliação histológica obtida pela biópsia transretal ${ }^{(5,6)}$, sendo este um procedimento geralmente seguro e bem tolerado pelos pacientes. Porém, alguns estudos apresentaram taxa de complicação de até $73 \%$, quando incluídos os eventos imediatos ou de resolução espontânea ${ }^{(7)}$. No Setor 
de Intervenção do Departamento de Diagnóstico por Imagem da Escola Paulista de Medicina - Universidade Federal de São Paulo (DDI/EPM-Unifesp), centro de referência que acolhe grande parte da população da zona sul da cidade de São Paulo, são realizadas cerca de 80 biópsias prostáticas por mês, perfazendo $36 \%$ do total de exames realizados no setor. Normalmente, as complicações após a biópsia de próstata por via transretal são leves e autolimitadas, incluindo hematúria, hematospermia, ou sangramento retal transitório. Às vezes, complicações mais graves como retenção urinária, evento infeccioso e sangramento retal importante podem ocorrer, necessitando tratamento adicional ${ }^{(8)}$.

O objetivo deste trabalho foi avaliar a incidência de complicações pós-procedimento nos pacientes submetidos a biópsia prostática transretal guiada por ultrassom no Setor de Intervenção do DDI/EPMUnifesp.

\section{MATERIAIS E MÉTODOS}

Foram avaliados, sequencialmente, 132 pacientes submetidos a biópsia prostática por via transretal, entre abril e julho de 2011, encaminhados ao nosso setor com suspeita clínica ou laboratorial de câncer de próstata. Todos foram submetidos ao mesmo protocolo de biópsia de próstata transretal guiada por ultrassom utilizado no nosso setor, após antibioticoterapia profilática com ciprofluoxacino. Após o posicionamento do paciente em decúbito lateral esquerdo com coxas fletidas, foi realizada a introdução do transdutor endocavitário acoplado ao guia de biópsia específico e seguiu-se a avaliação ultrassonográfica da próstata por via transretal. Posteriormente, foi realizada anestesia por bloqueio periprostático com $5 \mathrm{ml}$ de lidocaína a $2 \%$ diluída em $5 \mathrm{ml}$ de soro fisiológico, utilizando-se agulha de 18-gauge.

Após a anestesia, foi realizada a biópsia com retirada de 12 fragmentos representativos de toda a glândula, com agulha de 18-gauge, seguindo o padrão de sextantes preconizado pelo Colégio Brasileiro de Radiologia e Diagnóstico por Imagem e pela Sociedade Brasileira de Urologia. Fragmentos adicionais foram coletados na dependência de achados ultrassonográficos (lesões focais na zona periférica) ou conforme solicitação médica (achados palpatórios ou re-biópsia por saturação - 18 fragmentos). Os pacientes tiveram alta após realizar a primeira micção sem evidência de hematúria significativa depois do procedimento e receber as orientações quanto à ocorrência de complicações tardias.

Por contato telefônico, foi preenchido um questionário sobre eventos adversos, febris ou hemorrágicos, ocorridos até 14 dias após a biópsia prostática. No questionário esses eventos foram referidos como: hematúria, hematospermia ou hematoquezia, evento febril aferido, calafrio, disúria ou retenção urinária e suas respectivas evoluções e/ou tratamentos.

Dos 132 pacientes que realizaram biópsia nesse período, 29 foram excluídos em razão da impossibilidade de contato, após três tentativas em dias e horários diferentes, 4 por realizarem o procedimento durante internação hospitalar, 1 por morte de causas não relacionadas a biópsia (evento cardiovascular) e 1 por ter realizado o procedimento fora do protocolo estabelecido pelo setor ( sob sedação).

Para a avaliação das complicações, estas foram divididas em dois grupos: maiores e menores. Foram consideradas complicações maiores as que levaram o paciente a procurar serviço médico emergencial, após alta do nosso setor. Todas as outras complicações foram interpretadas como menores, autolimitadas, inerentes ao risco atribuído a um procedimento invasivo.

\section{RESULTADOS}

Os 97 pacientes selecionados apresentavam idades entre 43 e 83 anos (média de 66,2 anos). Destes, 38 (39,2\%) não apresentaram nenhum evento adverso após a biópsia prostática. Cinquenta e um pacientes $(52,6 \%)$ relataram pelo menos uma complicação menor, grande parte deles $(76,5 \%)$ referindo apenas um sintoma ou sinal isolado. Neste grupo, a complicação mais prevalente foi a hematúria macroscópica, referida por 39 pacientes. A duração da hematúria variou de 1 a 10 dias, apresentando média de 3,2 dias, sendo que $62,5 \%$ delas tiveram resolução espontânea nos três primeiros dias e apenas em 5\% dos casos persistiu por mais de uma semana.
A hematospermia foi o segundo sinal mais relatado, presente em $23,7 \%$ dos pacientes após a biópsia. A grande maioria desses pacientes $(82,6 \%)$ apresentou remissão espontânea da hematospermia e apenas quatro persistiam com o sintoma no momento do contato telefônico, porém não referiram necessidade de procurar atendimento médico por esse motivo.

Dois pacientes $(2 \%)$ referiram hematoquezia, ambos com resolução espontânea, um deles em um dia e outro em sete dias.

Em relação às complicações maiores, oito pacientes $(8,2 \%)$ necessitaram avaliação médica emergencial. Destes, sete $(87,5 \%)$ a procuraram por retenção urinária, com necessidade de passagem de sonda vesical, três deles necessitando de antibioticoterapia domiciliar associada.

Três pacientes $(3,1 \%)$ referiram febre, dois deles associada a disúria, com necessidade de passagem de sonda vesical e tratamento com antibioticoterapia domiciliar. Um dos pacientes que referiu febre aferida relatou resolução espontânea, sem necessidade de tratamento específico.

Apenas um paciente referiu hematúria persistente, associada a disúria, motivando-o a procurar avaliação médica hospitalar, tendo recebido antibioticoterapia em regime de internação. Porém, concomitantemente, referiu sintomas respiratórios, o que impossibilitou relacionar este evento diretamente com o procedimento. Outros cinco pacientes também referiram disúria, porém tiveram resolução espontânea, sem necessidade de avaliação médica adicional.

Calafrio foi o sintoma de menor prevalência, referido por apenas um paciente e estava associado a febre e retenção urinária.

A Tabela 1 relaciona as complicações relatadas pelos pacientes.

Tabela 1 Complicações tardias após a biópsia transretal da próstata guiada por ultrassonografia.

\begin{tabular}{ccc}
\hline & \multicolumn{2}{c}{$N^{\circ}$ de pacientes $(\%)$} \\
\cline { 2 - 3 } Sinais e sintomas & Presentes & Ausentes \\
\hline Hematúria & $39(40,2)$ & $58(59,8)$ \\
Hematospermia & $23(23,7)$ & $74(76,3)$ \\
Retenção urinária & $7(7,2)$ & $90(92,8)$ \\
Disúria & $6(6,2)$ & $91(93,8)$ \\
Febre & $3(3,1)$ & $94(96,9)$ \\
Hematoquezia & $2(2,1)$ & $95(97,9)$ \\
Calafrio & $1(1,0)$ & $96(99,0)$ \\
\hline
\end{tabular}




\section{DISCUSSÃO}

A detecção precoce do câncer de próstata é essencial para um melhor prognóstico, com consequente redução da morbidade dos pacientes portadores dessa afecção. Com o crescimento do rastreamento do câncer de próstata, tanto em serviços particulares como públicos, o número de biópsias de próstata transretais guiadas por ultrassom tende a aumentar, tornando indispensável ao radiologista intervencionista e ao médico solicitante o conhecimento das complicações inerentes ao método ${ }^{(9)}$.

Cinquenta e nove pacientes $(60,8 \%)$ do nosso estudo referiram pelo menos uma complicação tardia após a realização da biópsia prostática. Alguns outros estudos, como o de Miller et al., encontraram taxas menos significativas de complicações, chegando a $9,6 \%$, porém desconsideraram as complicações menores, autolimitadas ${ }^{(\mathbf{1 0})}$. Como não há uma padronização efetiva quanto à classificação das complicações, optamos por considerar a necessidade de avaliação médica adicional como definidora de complicação maior, pela sua repercussão socioeconômica. Assim sendo, obtivemos prevalência de 8,2\% complicações maiores e 52,6\% de complicações menores. Essas taxas são equivalentes a outros estudos da literatura internacional, como o de Rodríguez et al., que obtiveram prevalência semelhante $(63,6 \%)$ de complicações após o procedimento, sendo também a hematúria o principal sintoma encontrado por eles $(47,1 \%)^{(11)}$.

A hematúria macroscópica pós-biópsia é um evento geralmente autolimitado, bastante comum, referido por $40,2 \%$ dos pacientes incluídos no nosso estudo. Em estudo realizado em Rotterdam, Holanda, Rietbergen et al. avaliaram 1687 pacientes submetidos a biópsia prostática e encontraram prevalência semelhante $(45,3 \%)^{(\mathbf{1 2})}$. Por se tratar de um sintoma muito comum, alguns autores como Raaijmakers et al. consideraram complicação apenas os casos em que a hematúria persistiu por mais de três dias ${ }^{(13)}$, obtendo uma prevalência de $22,6 \%$. No presente estudo, apenas oito $(8,2 \%)$ pacientes se encaixariam nesse critério. A hematospermia foi o segundo sinal mais comumente encontrado, presente em $23,7 \%$ dos pacientes incluídos no nosso estudo, e nenhum deles foi considerado complicação maior. Na literatura há grande variação - de 9,1\% a 78,3\% - quanto à prevalência da hematospermia após a biópsia prostática $^{(11,14)}$. A maior dessas prevalências $(78,3 \%)$ foi obtida por Peyromaure et al., que consideraram apenas a relação entre hematospermia e pacientes que referiram atividade sexual no período avaliado ${ }^{(\mathbf{1 4})}$. Quando relacionada com o total de pacientes, foi encontrada prevalência de $54,9 \%$, taxa ainda muito superior à registrada no presente estudo.

Geralmente associados a infecção do trato urinário (ITU), disúria, febre ou calafrio foram referidos por 10 pacientes do nosso estudo, porém, destes, apenas 3 $(3,1 \%)$ foram caracterizados como eventos maiores. A ITU é considerada a segunda complicação mais frequente da biópsia de próstata, atrás apenas das complicações hemorrágicas. Apesar de simples, a ITU ocorre com certa frequência após a biópsia de próstata e ITUs febris não são incomuns após o procedimento ${ }^{(15,16)}$. Sepse é uma das complicações mais graves, porém rara, e é encontrada em menos de $2 \%$ dos casos $^{(12)}$. Em nosso serviço, em levantamento realizado desde 2007, apenas um paciente necessitou de internação em unidade de terapia intensiva por sepse após biópsia de próstata, recebendo alta após 14 dias de tratamento. Estudos comprovaram bacteriúria assintomática e bacteremia transitória em $16 \%$ a $100 \%$ dos casos após a realização do procedimento ${ }^{(17,18)}$, sendo consenso, atualmente, a importância da antibioticoterapia profilática no preparo do paciente antes da biópsia, no intuito de manter baixos os índices de complicações decorrentes desse procedimento ${ }^{(19,20)}$. Entretanto, muitos estudos têm apontado para a falta de um algoritmo padrão a ser seguido nesse preparo ${ }^{(21)}$.

Apesar de termos registrado taxa de complicações maiores $(8,2 \%)$ equiparável à maioria dos serviços que se dedicam a este estudo utilizando critérios semelhantes ${ }^{(22)}$, tivemos incidência elevada de retenção urinária quando comparada com trabalhos similares na literatura (Tabela 2). Entre os fatores de risco de retenção urinária na população geral, vários estão presentes nos pacientes submetidos a biópsia: obstrução prévia por aumento do volume da prós- tata ou pela potencial obstrução pela presença de hematúria, infecção concomitante, particularmente prostatites, e uso de medicamentos com propriedades anticolinérgicas (antidepressivos tricíclicos, alfa-adrenérgicos e anti-inflamatórios não hormonais). Algumas condições presentes no pósoperatório de cirurgias se assemelham às do procedimento de biópsia, por exemplo, a ocorrência de dor ou de procedimento anestésico $^{(23)}$. Na nossa experiência, a alta do Setor de Intervenção ser efetivada apenas após a primeira micção clara seria o bastante para prevenir esta complicação. Este achado, além estimular a atenção redobrada neste critério de alta, mostra a necessidade de outros estudos que pesquisem suas possíveis causas e indiquem novos critérios ou medidas adicionais, no intuito de reduzir ao máximo essas complicações.

Tabela 2 Revisão literária da retenção urinária na biópsia transretal da próstata guiada por ultrassonografia.

\begin{tabular}{cc}
\hline Referência & $\begin{array}{c}\text { Retenção } \\
\text { urinária }\end{array}$ \\
\hline${\text { Jeon et al. }{ }^{(7)}}^{(11)}$ & $1,1 \%$ \\
Rodríguez et al. & $1,6 \%$ \\
Rietbergen et al. ${ }^{(12)}$ & $0,4 \%$ \\
Raaijmakers et at. ${ }^{(13)}$ & $0,4 \%$ \\
Djavan et al. ${ }^{(15)}$ & $2,6 \%$ \\
Lee et al. ${ }^{(2)}$ & $1,7 \%$ \\
Solha et al. (presente estudo) & $7,2 \%$ \\
\hline
\end{tabular}

\section{CONCLUSÃO}

Concluímos que, apesar de a taxa de retenção urinária referida pelos pacientes do presente estudo ser maior que a média dos trabalhos citados, a prevalência de complicações maiores e menores no nosso serviço se equipara aos dos que se dedicaram a este estudo, corroborando a segurança do procedimento e a baixa incidência de complicações tardias maiores.

\section{REFERÊNCIAS}

1. Jemal A, Siegel R, Ward E, et al. Cancer statistics, 2008. CA Cancer J Clin. 2008;58:71-96.

2. Ministério da Saúde. Instituto Nacional de Câncer. Câncer de próstata. In: Estimativa 2010. Incidência de câncer no Brasil. Rio de Janeiro, RJ: INCA; 2009.

3. Kelloff GJ, Choyke P, Coffey DS; Prostate Cancer Imaging Working Group. Challenges in clinical prostate cancer: role of imaging. AJR Am J Roentgenol. 2009;192:1455-70. 
4. Kundra V, Silverman PM, Matin SF, et al. Imaging in oncology from the University of Texas $\mathrm{M}$. D. Anderson Cancer Center: diagnosis, staging, and surveillance of prostate cancer. AJR Am J Roentgenol. 2007;189:830-44.

5. Goldman SM. Ressonância magnética da prós tata. Radiol Bras. 2009;42(3):vii-viii.

6. Santos VCT, Milito MA, Marchiori E. O papel atual da ultra-sonografia transretal da próstata na detecção precoce do câncer prostático. Radiol Bras. 2006;39:185-92.

7. Jeon SS, Woo SH, Hyun JH, et al. Bisacodyl rectal preparation can decrease infectious complications of transrectal ultrasound-guided prostate biopsy. Urology. 2003;62:461-6.

8. Berger AP, Gozzi C, Steiner H, et al. Complication rate of transrectal ultrasound guided prostate biopsy: a comparison among 3 protocols with 6 , 10 and 15 cores. J Urol. 2004;171:1478-80.

9. Castro HAS, Iared W, Shigueoka DC, et al. Contribuição da densidade do PSA para predizer o câncer da próstata em pacientes com valores de PSA entre 2,6 e 10,0 ng/ml. Radiol Bras. 2011;44: 205-9.

10. Miller J, Perumalla C, Heap G. Complications of transrectal versus transperineal prostate biopsy. ANZ J Surg. 2005;75:48-50.
11. Rodríguez LV, Terris MK. Risks and complications of transrectal ultrasound guided prostate needle biopsy: a prospective study and review of the literature. J Urol. 1998;160(6 Pt 1):2115-20.

12. Rietbergen JB, Kruger AE, Kranse R, et al. Complications of transrectal ultrasound-guided systematic sextant biopsies of the prostate: evaluation of complication rates and risk factors within a population-based screening program. Urology. 1997;49:875-80.

13. Raaijmakers R, Kirkels WJ, Roobol MJ, et al. Complication rates and risk factors of 5802 transrectal ultrasound-guided sextant biopsies of the prostate within a population-based screening program. Urology. 2002;60:826-30.

14. Peyromaure M, Ravery V, Messas A, et al. Pain and morbidity of an extensive prostate 10-biopsy protocol: a prospective study in 289 patients. J Urol. 2002;167:218-21.

15. Djavan B, Waldert M, Zlotta A, et al. Safety and morbidity of first and repeat transrectal ultrasound guided prostate needle biopsies: results of a prospective European prostate cancer detection study. J Urol. 2001;166:856-60.

16. Aus G, Ahlgren G, Bergdahl S, et al. Infection after transrectal core biopsies of the prostate - risk factors and antibiotic prophylaxis. $\mathrm{Br} \mathrm{J}$ Urol. 1996;77:851-5.
17. Crawford ED, Haynes AL Jr, Story MW, et al Prevention of urinary tract infection and sepsis following transrectal prostatic biopsy. J Urol. 1982;127:449-51

18. Lindert KA, Kabalin JN, Terris MK. Bacteremia and bacteriuria after transrectal ultrasound guided prostate biopsy. J Urol. 2000;164:76-80.

19. Aron M, Rajeev TP, Gupta NP. Antibiotic prophylaxis for transrectal needle biopsy of the prostate a randomized controlled study. BJU Int. 2000;85: 682-5.

20. Kapoor DA, Klimberg IW, Malek GH, et al. Single-dose oral ciprofloxacin versus placebo for prophylaxis during transrectal prostate biopsy. Urology. 1998;52:552-8.

21. Davis M, Sofer M, Kim SS, et al. The procedure of transrectal ultrasound guided biopsy of the prostate: a survey of patient preparation and biopsy technique. J Urol. 2002;167(2 Pt 1):566-70.

22. Lee SH, Chen SM, Ho CR, et al. Risk factors associated with transrectal ultrasound guided prostate needle biopsy in patients with prostate cancer. Chang Gung Med J. 2009;32:623-7.

23. Selius BA, Subedi R. Urinary retention in adults: diagnosis and initial management. Am Fam Physician. 2008;77:643-50. 